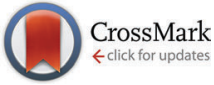

Cite this: J. Mater. Chem. B, 2015, 3, 8902

Received 24th August 2015, Accepted 19th October 2015

DOI: $10.1039 / c 5 t b 01737 h$

www.rsc.org/MaterialsB

\section{Glycosaminoglycan functionalization of mechanically and topologically defined collagen I matrices}

\author{
Liv Kalbitzer, ${ }^{a}$ Katja Franke, ${ }^{a}$ Stephanie Möller, ${ }^{b}$ Matthias Schnabelrauch ${ }^{b}$ and \\ Tilo Pompe*a
}

\begin{abstract}
Collagen I and glycosaminoglycans (GAGs) are major components of the extracellular matrix in mammals and widely used for in vitro cell culture matrices. While composition, network microstructure and mechanics of these matrices sensitively determine cell fate, they are hard to adjust independently during matrix reconstitution. We report on a sequential preparation procedure of collagen I matrices, which allows a defined adjustment of network topology and mechanics in combination with GAG functionalization. Collagen I solution concentrations of 1.5 to $7 \mathrm{mg} \mathrm{ml}^{-1}$ allowed to vary topology (pore size) and elasticity of resulting networks with Young's moduli of 5 to $220 \mathrm{~Pa}$. Zero-length crosslinking using carbodiimide chemistry increased Young's modulus 3 to 5 times without changing network topology. An optional covalent binding of hyaluronan and synthetically sulfated hyaluronan to the preformed matrices led to topologically unaffected networks with a stable functionalization with $30 \mu \mathrm{g}$ GAG per mg collagen. While sulfated GAGs were stably attached to collagen I networks via physisorption or covalent binding at neutral and acidic conditions, non-sulfated hyaluronan required acidic conditions and covalent binding for stable attachment. In conclusion, this approach provides options to independently adjust biophysical and biochemical parameters of collagen I networks for in vitro studies.
\end{abstract}

\section{Introduction}

Tissue growth, pathology and regeneration are controlled by the interplay of the involved cells and microenvironmental cues. In this context, the biophysical and biochemical properties of the extracellular matrix (ECM) play a pivotal role in controlling cell adhesion, growth, differentiation, migration and apoptosis. ${ }^{1-6}$ The complex interplay of ECM parameters frequently limits a clear distinction between their individual contributions to cell behavior, which is especially true for the in vivo situation. Furthermore, the applicability of in-depth analytical techniques is limited in vivo. To circumvent these problems, biomimetic matrices represent a promising approach to conduct in vitro experiments in defined microenvironments to better understand the influence and interplay of specific ECM parameters e.g. topology, elasticity and composition. Such setups allow the investigation of cell-ECM interactions with high-end analytical tools ranging from single cell gene analysis, optical nanoresolution to single cell tracking. Furthermore, the development

\footnotetext{
${ }^{a}$ Institute of Biochemistry, Faculty of Biosciences, Pharmacy and Psychology, Universität Leipzig, Leipzig 04103, Germany. E-mail: tilo.pompe@uni-leipzig.de; Fax: +49 34197 36939; Tel: +49 3419736931

${ }^{b}$ Biomaterials Department, Innovent e.V., o7745 Jena, Germany
}

and usage of advanced biomimetic microenvironments will increase the relevance of in vitro studies for understanding physiological processes and has the potential to reduce animal experiments.

In order to develop in vitro ECM models, the control of the full range of biochemical as well as biophysical properties of the ECM is of outstanding importance. Next to composition and mechanics, the three-dimensional (3D) character of the ECM microenvironment is increasingly recognized as an important parameter to control receptor activation, cell shape and dynamics as well as many downstream signaling pathways. ${ }^{7-10}$ Matrices reconstituted from native components of the ECM have been shown to be well-suited models as they resemble the fibrillar topology of in vivo ECM networks and provide highly specific binding sites for many cell surface receptors and soluble mediators. ${ }^{11,12}$ Alternative systems based on synthetic and biohybrid hydrogels are also discussed as promising approaches, ${ }^{10,13}$ but they usually lack important topological characteristics of fibrillar ECM networks.

Collagen I (Coll I) based networks are among the most prominent approaches to reconstitute 3D ECM in vitro. The main reasons for the broad usage of Coll I are its high abundance in the ECM of mammals and its ability to easily self-assemble into fibrillar networks under in vitro conditions. 
Characteristics of such networks are modulated by conditions during the fibrillation process including temperature, $\mathrm{pH}$ and concentrations of salt and buffer ions, proteins (including other collagen types), and polysaccharide supplements. ${ }^{14-19}$ Variation of these factors results in differences in network porosity, fibril length and diameter, fibril and network elasticity as well as nanoscale order of tropocollagen building blocks. Additionally, zero-length or non-zero-length crosslinking via carbodiimides or glutaraldehyde, respectively, are used to modulate mechanics of Coll I networks. ${ }^{20,21}$ In order to prepare well defined Coll I networks all these parameters need to be tightly controlled.

Proteoglycans and especially their major functional building blocks, the glycosaminoglycans (GAGs), are considered as additional important components of in vitro matrices because they are known to play a major role for the in vivo function of the ECM. They affect many physiological and pathological processes, e.g. wound healing, tumor growth and cancer cell migration. $^{22-25}$ In vivo, sulfated GAGs, like heparan sulfate and the non-sulfated GAG, hyaluronan (HA), contribute to important functions of the ECM including water homeostasis, lubrication and cushioning as well as binding, release and presentation of cytokines and other soluble mediators. Furthermore, GAGs are ligands for specific cell surface receptors, e.g. HA for CD44 and heparan sulfate for L-selectin. ${ }^{26-28}$ Because of that, several approaches have been applied to incorporate GAGs into reconstituted matrices also in the context of Coll I based fibrillar matrices. Commonly Coll I/GAG matrices are prepared by mixing Coll I and GAG in solution prior to fibrillation. ${ }^{29-33}$ However, the presence of GAGs in Coll I solutions is reported to have a strong influence on fibril length, diameter, mechanics and nanostructure. ${ }^{34-36}$ Hence, the sensitivity of the fibrillation process to the composition of the Coll I solution hinders the precise control of network parameters when adding supplemental components in varying amounts or comparing networks with different supplements, e.g. GAGs with different degree or pattern of sulfation. As a consequence, it is difficult to prepare Coll I matrices at predefined topology and mechanics with and without GAG presence. However, such a distinct and quantitative modulation of ECM parameters is needed to correlate them to specific cell responses.

In order to circumvent these shortcomings, we used a sequential preparation approach to vary topology and mechanics of Coll I networks independently of GAG functionalization. We compared a set of networks with different Coll I concentrations and with and without chemical crosslinking by $N$-(3-dimethylaminopropyl)- $N^{\prime}$ ethylcarbodiimide (EDC) and demonstrated the optional GAG functionalization by covalent binding of non-sulfated HA (HA) and highly sulfated HA (hsHA).

\section{Experimental}

\section{HA synthesis and chemical modification}

Native high molecular weight HA (from Streptococcus, weight average molecular weights as determined with laser light
Table 1 Characteristics of synthesized HA derivatives degree of sulfation (D.S.), number average $\left(M_{n}\right)$ and weight average $\left(M_{w}\right)$ molecular weights as determined by laser light scattering detection and refraction detection (in brackets), molecular weight distributions (polydispersity index - PD) based on the values calculated from refraction detection

\begin{tabular}{lll}
\hline Sample & HA & hsHA \\
\hline D.S. & - & 3.8 \\
$M_{\mathrm{n}} /\left(\mathrm{g} \mathrm{mol}^{-1}\right)$ & $15435(40105)$ & $12285(26745)$ \\
$M_{\mathrm{w}} /\left(\mathrm{g} \mathrm{mol}^{-1}\right)$ & $23040(87570)$ & $21425(42830)$ \\
$\mathrm{PD}$ & 2.18 & 1.60
\end{tabular}

scattering $M_{\mathrm{w}}=1.1 \times 10^{6} \mathrm{~g} \mathrm{~mol}^{-1}$, polydispersity index $\mathrm{PD}=4.8$ ) was obtained from Aqua Biochem (Dessau, Germany), sulfur trioxide/dimethylformamide complex ( $\mathrm{SO}_{3}-\mathrm{DMF}$, purum, $\geq 97 \%$, active $\mathrm{SO}_{3} \geq 48 \%$ ) from Fluka Chemie (Buchs, Switzerland). Fluorescence marker (ATTO $565-\mathrm{NH}_{2}$ ) was purchased from ATTO-TEC (Siegen, Germany).

The highly sulfated HA derivative (hsHA) was synthesized and characterized as described previously. ${ }^{37}$ Low molecular weight HA was prepared by ozonolysis of high molecular weight native HA. A 1\% aqueous solution of high molecular weight HA was treated with ozone, prepared with an ozone generator COM-AD-02 (ANSEROS Klaus Nonnenmacher, Tübingen, Germany) for $2 \mathrm{~h}$. The ozone concentration amounted to approx. $30 \mathrm{~g} \mathrm{~m}^{-3}$ and a flow rate of 20-30 $\mathrm{l} \mathrm{h}^{-1}$ was used. Finally, $\mathrm{N}_{2}$ was passed through the solution for $30 \mathrm{~min}$ to expel free ozone. The remaining clear solution was dialyzed against distilled water, lyophilized and dried in vacuum. The HA was obtained with $75-85 \%$ yield. Analytical data of the HA derivatives (HA, hsHA) are summarized in Table 1.

The functionalization of the HA derivatives (HA, hsHA) with fluorescence dye (ATTO 565- $\mathrm{NH}_{2}$ ) was carried out at the reducing end-group of the macromolecule using the following procedure: $0.5 \mathrm{mmol}$ of $\mathrm{HA}$ and $0.25 \mathrm{mmol}$ of hsHA, respectively, were dissolved in $30 \mathrm{ml}$ of distilled water and the $\mathrm{pH}$ value was adjusted with $0.1 \mathrm{M} \mathrm{NaOH}$ to $7.5-8$. Then, $500 \mu \mathrm{g}(0.6 \mu \mathrm{mol})$ of the fluorescence marker, dissolved in water, were added to the solution and the reaction mixture was stirred for $6 \mathrm{~h}$ at room temperature. After this time, the $\mathrm{pH}$ of the mixtures was adjusted to 7.5 and $\mathrm{NaCNBH}_{3}$ was added in two steps. After stirring for 3 days at room temperature, the reaction mixture was first dialyzed against deionized water at $\mathrm{pH}$ 8-8.5 and afterwards dialysis was continued against deionized water at $\mathrm{pH} 5.5$ to remove residual unbound dye. After filtration, lyophilization and drying in vacuum, the labeled GAGs were obtained with $85 \%$ yield.

\section{Preparation of Coll I networks}

Coll I networks were prepared on glass coverslips (13 mm, VWR international, Leuven, Belgium) which were functionalized with a maleic anhydride copolymer for covalent immobilization of Coll I on the substrate as described elsewhere. ${ }^{38}$ Briefly, thin films of $0.14 \mathrm{wt} \%$ poly(styrene-alt-maleic anhydride) (PSMA) (AppliChem, Darmstadt, Germany) ( $\left.M_{\mathrm{w}} 20000-30000\right)$ in acetone/THF (1:2, AppliChem) were spincoated on aminosilane (VWR international) functionalized glass coverslips and 
tempered at $120{ }^{\circ} \mathrm{C}$ for $2 \mathrm{~h}$. After washing in acetone, coverslips were stored up to 3 months and were tempered at $120{ }^{\circ} \mathrm{C}$ for $2 \mathrm{~h}$ before preparing the Coll I networks. Covalent binding of the lysine side chains of the Coll I to the anhydride groups of the polymer leads to a stable immobilization of the network. To prepare networks with different concentrations of collagen, two stock solutions of rat tail Coll I were used, one with $3.37 \mathrm{mg} \mathrm{ml} \mathrm{m}^{-1}$ (BD Bioscience, Heidelberg, Germany) and one with $8.87 \mathrm{mg} \mathrm{ml}^{-1}$ (Corning, NY). Neutralized Coll I solutions ( $\mathrm{pH}$ 7.4) were prepared by mixing 8 parts of Coll I stock solution with 1 part of sodium hydroxide (0.1 M, Diagonal, Münster, Germany) and 1 part of $10 \times$ phosphate buffered saline (PBS, Biochrom, Berlin, Germany). Final Coll I concentrations between 1.5 to $7 \mathrm{mg} \mathrm{ml}{ }^{-1}$ were achieved by diluting the solution with $1 \times$ PBS. Solutions were prepared on ice and immediately used for network reconstitution. A volume of $20 \mu \mathrm{l}$ was placed on freshly tempered PSMA coverslips and fibrillogenesis was initialized by warming up to $37{ }^{\circ} \mathrm{C}$ for $90 \mathrm{~min}$ and 95\% relative humidity. Networks were strictly kept in a hydrated state to avoid collapsing of the 3D network structure. With this approach, network layers of roughly $150 \mu \mathrm{m}$ in thickness are prepared as previously shown. ${ }^{39}$

\section{EDC crosslinking of networks}

Coll I networks were prepared at solution concentrations of 2, 4 and $5.5 \mathrm{mg} \mathrm{ml}^{-1}$ as described above. Subsequently, networks were incubated in a freshly prepared solution of EDC (Sigma Aldrich, Munich, Germany) for $2 \mathrm{~h}$ at room temperature. EDC concentrations of $2 \mathrm{mM}\left(0.4 \mathrm{mg} \mathrm{ml}^{-1}\right), 20 \mathrm{mM}\left(4 \mathrm{mg} \mathrm{ml}^{-1}\right), 50 \mathrm{mM}$ $\left(10 \mathrm{mg} \mathrm{ml}^{-1}\right), 100 \mathrm{mM}\left(20 \mathrm{mg} \mathrm{ml}^{-1}\right)$, and $500 \mathrm{mM}\left(100 \mathrm{mg} \mathrm{ml}^{-1}\right)$ in either PBS (pH 7.4) or 2-( $N$-morpholino)ethanesulfonic acid (MES, 0.1 M, pH 6, Sigma Aldrich) were used. After washing, networks were stored in PBS up to $48 \mathrm{~h}$ until characterization.

\section{Mechanical characterization of networks}

For micromechanical characterization of networks, the Young's modulus, $E$, was determined. It was calculated by fitting forcedistance curves recorded by colloidal probe spectroscopy using the Hertz model. These measurements were performed with a Nanowizard III (JPK Instruments, Berlin, Germany) and the probes were prepared by attaching a $15 \mu \mathrm{m}$ polystyrene microbead (Polyscience Europe GmbH, Eppelheim, Germany) to a tipless MLCT triangular cantilever with a nominal spring constant of $\sim 60 \mathrm{nN} \mathrm{m}^{-1}$ (Bruker AFM probes, Camarillo, CA). The exact spring constant was determined by the thermal noise method. ${ }^{40}$ All samples were characterized in PBS at room temperature. A minimum of 45 force-distance curves with an indentation of at least $5 \mu \mathrm{m}$ was measured for each sample.

\section{Topological characterization of networks}

Coll I networks were stained with 5-(and-6)-carboxytetramethylrhodamine succinimidyl ester (5(6)-TAMRA-SE, Biotium, Hayward, CA) for topological analysis. To do so, the networks were incubated in freshly prepared 5(6)-TAMRA-SE solution (50 $\mu \mathrm{M}$ in PBS, $1 \mathrm{~h}, \mathrm{RT}$ ), washed in PBS and embedded on coverslips using Eukitt ${ }^{\circledR}$ (Diagonal). Images of Coll I networks were taken using a confocal laser scanning microscope (cLSM)
LSM700 (Zeiss, Jena, Germany) with a $63 \times /$ NA 1.3 water immersion objective. Images were acquired with 8-bit color depth, $1024 \times 1024$ pixels in resolution and a vertical stack size of 20 images at $5 \mu \mathrm{m}$ distance (equivalent to $100 \mu \mathrm{m}$ ). The voxel size of the acquired images was $0.1 \times 0.1 \times 0.5 \mu \mathrm{m}(x \times y \times z)$. Pore size and fibril diameter were analyzed using a home-built image processing procedure as described by Franke et al. ${ }^{39}$ The topological analysis was performed with at least 3 positions per sample and 3 samples per condition.

\section{GAG modification of networks and quantification of gag content}

GAGs were either physisorbed or covalently bound to Coll I networks prepared from a Coll I solution of $2 \mathrm{mg} \mathrm{ml} \mathrm{ml}^{-1}$. Fluorescently labeled GAGs (HA-ATTO 565, hsHA-ATTO 565) were used to verify GAG binding, its homogeneous distribution and to quantify GAG amount. GAG physisorption and covalent binding via EDC was investigated at neutral (PBS, $\mathrm{pH} 7.4$ ) and acidic (MES, pH 5 \& 6) conditions. In preliminary experiments different GAG concentrations $\left(c_{\mathrm{GAG}}=0.05-1 \mathrm{mg} \mathrm{ml}^{-1}\right)$ were tested. A concentration $>0.1 \mathrm{mg} \mathrm{ml}^{-1}$ did not lead to a higher amount of stably bound GAG after 1 day, but to a higher release during the first hours. Thus a GAG concentration of $0.1 \mathrm{mg} \mathrm{ml}^{-1}$ was chosen for further experiments.

For physisorption, Coll I networks were incubated in a solution of $\mathrm{HA}$ or hsHA for $2 \mathrm{~h}$ at room temperature. The networks were washed with PBS, incubated in PBS overnight and washed again. For covalent binding, networks were at first incubated in GAG solution as described above. After removal of GAG solution, a freshly prepared solution of EDC $(20 \mathrm{mM}$ in MES, 0.1 M, pH 5) was added and incubated for $2 \mathrm{~h}$ at room temperature. Afterwards, networks were washed in PBS, incubated in PBS overnight and washed again.

Visual inspection of GAG binding and distribution throughout the network was performed via cLSM, see above. For GAG quantification by fluorimetry, networks were prepared as described above and incubated in a GAG solution of $0.1 \mathrm{mg} \mathrm{ml}^{-1}$ in MES $(0.1 \mathrm{M}$, pH 5). Next, networks modified with HA-ATTO 565 and hsHAATTO 565 were digested in a papain solution $\left(0.02 \mu \mathrm{g} \mathrm{ml}^{-1}\right.$ papain from papaya latex, $10 \mathrm{mM}$ EDTA, $5 \mathrm{mM}$-cysteine (all Sigma Aldrich) in $5 \times$ PBS) for $2 \mathrm{~h}$ at $60{ }^{\circ} \mathrm{C}$. Fluorescence intensity of fully digested networks was measured with a plate reader (Infinite 200 PRO, Tecan, Männedorf, Switzerland) and analyzed using calibration curves. Concentrations were determined at day 1, 5 and 12 of incubation in PBS buffer with supplements of $1 \%$ bovine serum albumin (Sigma Aldrich). GAG amount was related to Coll I amount of the network with $40 \mu \mathrm{g}$ Coll I per sample taken from our previous analysis. $^{39}$

\section{Results and discussion}

Within this work we developed and characterized 3D Coll I matrices. We set up a stepwise preparation approach to independently and precisely adjust pore size, elasticity and GAG 


\section{Network formation}

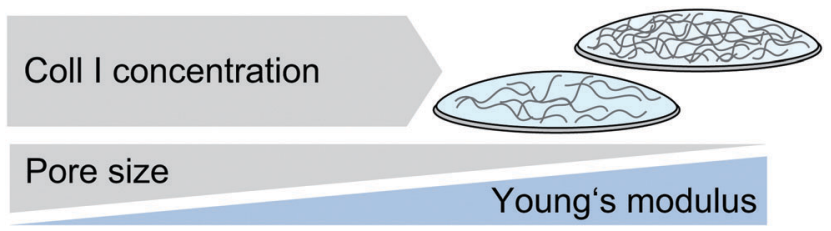

2. Network modification

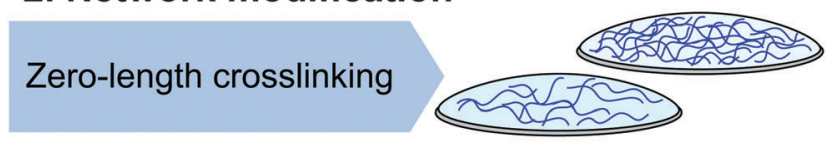

Young's modulus

GAG-binding

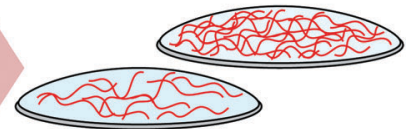

Fig. 1 Modulation of GAG functionalization of Coll I network independent of topology and mechanics.

functionalization of Coll I networks (Fig. 1). This combinatorial approach is intended to be used in in vitro cell culture studies on the impact of specific ECM properties.

\section{Elasticity and topology of Coll I networks}

At first we investigated the elasticity of Coll I networks in dependence on Coll I concentration during fibril formation. Furthermore, the change of network properties after crosslinking via EDC was evaluated. Based on this set of characterized networks, varied in topology and elasticity, a subsequent GAG functionalization was studied as an optional modification.

By increasing Coll I concentration from 1.5 to $7.0 \mathrm{mg} \mathrm{ml}^{-1}$, we observed a monotone increase from a low (5 Pa) to a high Young's modulus $(E)$ (220 Pa) of Coll I matrices (Fig. 2A). The increase of $E$ with increasing Coll I concentration, $c$, corresponds with the decreased pore size at higher Coll I concentrations. For the scaling exponent of $E \sim c^{x}$, we found $x=1.7$, which compares well to values from previous studies and model predictions. ${ }^{41-44}$ In the light of the detailed modelling of crosslinked semiflexible polymer networks by Kurniawan et al. ${ }^{44}$ this value suggests, that in the linear elastic regime, Coll I network mechanics are dominated by the entanglement of semiflexible fibrils rather than by crosslinking between fibrils. This conclusion will be further used in the discussion on the modification of Coll I networks' elasticity by crosslinking below. The large variation of $E$ for a high Coll I concentration can be attributed to a large heterogeneity in pore size, which might result from locally hindered transport of tropocollagen and microfibrils during fibril formation at high Coll I concentrations.

The modification of existing Coll I networks with EDC zerolength crosslinking is a well-known process, however, the precise control of preparation conditions is not well documented in literature. EDC crosslinking affects network elasticity in dependence on $\mathrm{pH}$ and EDC concentration. We observed a slight increase of $E$ for $\mathrm{pH} 7.4$ and for $\mathrm{pH} 6$ at low concentrations of EDC ( $2 \mathrm{mM})$ compared to unmodified networks. $E$ markedly increased after crosslinking at $\mathrm{pH} 6$ with EDC concentrations larger than $20 \mathrm{mM}$ (Fig. 2B and C). For the $\mathrm{pH}$ dependency of the EDC reaction different relations are reported in literature. ${ }^{45,46}$ However, we found a higher efficiency of crosslinking at more acidic conditions, which is reasonable due to the higher degree of protonation at low $\mathrm{pH}$. EDC as well as Coll I concentration were important for the resulting change of $E$. Increasing EDC concentration from $20 \mathrm{mM}$ to $500 \mathrm{mM}$ led to 3 to 5 times larger Young's moduli compared to unmodified networks $\left(c_{\text {Coll I }}=\right.$ $2 \mathrm{mg} \mathrm{ml}^{-1}$; Fig. 2B). These results are in part contradicting to previous studies which showed that a saturation of crosslinking is already achieved at EDC concentrations of $20 \mathrm{mM}^{47}$ Furthermore, crosslinking (at $c_{\mathrm{EDC}}=20 \mathrm{mM}$ ) was observed to have a higher impact on $E$ at high Coll I concentrations (Fig. 2C) than at low Coll I concentrations. We also verified that network topology was unaffected by EDC crosslinking (Fig. 2D). This is expected as EDC only acts intrafibrillarly, which means only tropocollagen inside microfibrils are crosslinked but no linking between different fibrils occurs. ${ }^{21,48,49}$ This is also important with respect to the discussion of mechanical network models, see above. The increase in $E$ by EDC crosslinking has to be attributed to an increase in stiffness of the entangled semiflexible Coll I fibrils, and again a scaling of $E \sim c^{x}$ with $x \approx 1.5$ is found in our data of crosslinked Coll I networks, as expected (Fig. 2C).

As outlined above, we verified two tools to independently adjust network elasticity: variation of Coll I concentration and zero-length crosslinking by EDC. An increased $E$ is either attributed to decreased pore sizes (higher Coll I concentration) or to an increased stiffness of fibrils (zero-length crosslinking). Using a combination of these methods one is able to form a set of matrices with (i) same pore size and different $E$ (unmodified and crosslinked network), (ii) different pore size and different $E$ (different Coll I concentrations) and (iii) different pore size and similar $E$ (unmodified network with high Coll I concentration compared to crosslinked network with low Coll I concentration). These defined and adjustable matrices are an important base to investigate the impacts of pore size, fibril stiffness and network elasticity on cell behavior.

\section{GAG modification of Coll I networks}

In a second set of experiments we investigated strategies for a subsequent modification of Coll I networks $\left(c_{\text {Coll I }}=2 \mathrm{mg} \mathrm{ml}^{-1}\right)$ with GAGs with and without sulfate groups, namely HA and highly sulfated HA (hsHA) of similar molecular weight. We used a GAG concentration of $0.1 \mathrm{mg} \mathrm{ml}{ }^{-1}$, as deduced from initial screening experiments, to achieve saturation of Coll I networks with GAGs.

Low molecular weight hsHA $\left(M_{\mathrm{w}}=21 \mathrm{kDa}\right.$, D.S. $\left.=3.8\right)$ was synthesized and characterized as described previously. ${ }^{31,37}$ Non-sulfated low molecular weight HA with similar molecular weight $\left(M_{\mathrm{w}}=23 \mathrm{kDa}\right)$ was prepared by controlled ozonolysis of native high molecular weight HA. ${ }^{13} \mathrm{C}-\mathrm{NMR}$ investigations of the degraded HA did not show any structural changes compared to 

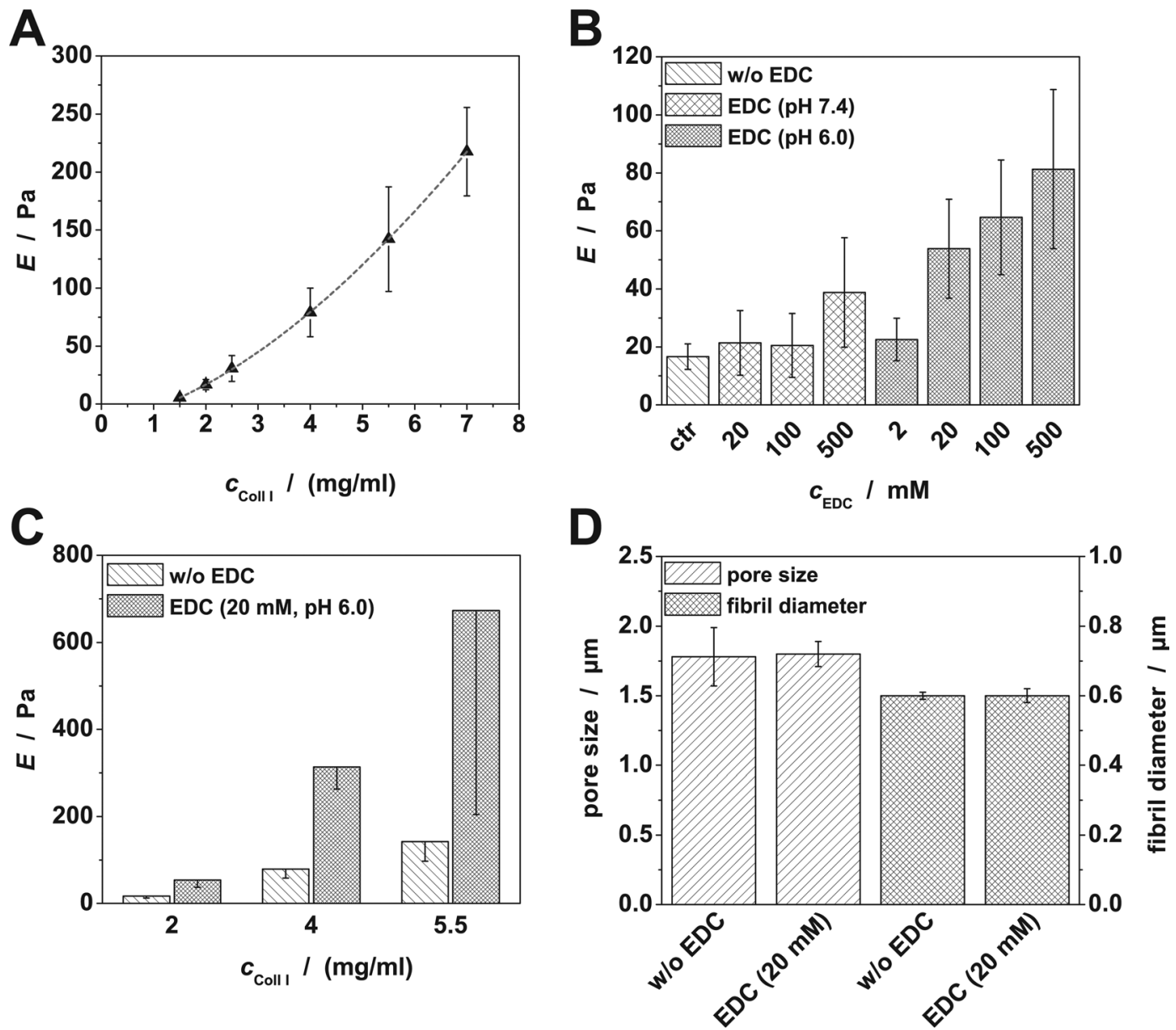

Fig. 2 Mechanical and topological characterization of Coll I networks without and with zero-length crosslinking via EDC. (A) $E$ was determined in dependence on Coll I concentration $c_{\text {Coll }}$ in a range from 1.5 to $7 \mathrm{mg} \mathrm{ml}^{-1}$. The dashed line indicates a power law fit with $E \sim c_{\text {Colll. }}^{1.7}$ (B) $E$ was determined in dependence on EDC concentration and $\mathrm{pH}$ value for Coll I networks prepared from a solution of $c_{\text {Colll }}=2 \mathrm{mg} \mathrm{ml}^{-1}$. (C) Comparison of $E$ of Coll I networks without and with crosslinking for various Coll I concentrations. (D) Topological analysis of Coll I networks without and with crosslinking ( $c_{\text {CollI }}=$ $2 \mathrm{mg} \mathrm{ml}^{-1}, C_{\mathrm{EDC}}=20 \mathrm{mM}, \mathrm{pH}$ 6). Error bars indicate \pm standard deviation (S.D.). (In (C) only - S.D. is shown.) Mean values were obtained from three independent experiments $(n=3)$ with 45 measurements per sample.

native HA (data not shown). The functionalization of HA derivatives (HA, hsHA) with an amino group containing the fluorescence marker ATTO $565-\mathrm{NH}_{2}$ was carried out at the reducing end-group of the GAGs. The formed imine linker structure with a $\mathrm{C}=\mathrm{N}$ double bond was reduced with sodium cyanoborohydride $\left(\mathrm{NaCNBH}_{3}\right)$ to obtain the more stable amine linker.

The degree of sulfation does not only affect physiological function, but it is also expected to lead to a differential binding of GAG to Coll I networks..$^{35,36,50}$ We used subsequent and combined steps of GAG physisorption and covalent binding to preformed Coll I networks in order to find optimal conditions for GAG modification. Initial experiments showed different binding behavior of HA and hsHA depending on $\mathrm{pH}$ during physisorption and the subsequent covalent binding step via EDC (Table 2). As discussed above, a low pH was expected to improve EDC crosslinking, but our results also showed an impact of $\mathrm{pH}$ on physisorption. Strong physisorption of hsHA and negligible physisorption of HA at neutral buffer conditions were observed, in accordance to previous findings. ${ }^{51}$ (Data in Table 2 corroborate these results also for HA physisorption at

Table 2 GAG binding at different $\mathrm{pH}$ value during physisorption and subsequent EDC crosslinking (PBS at pH 7.4, MES at pH 5 \& 6). Coll I networks were first incubated in the GAG solution for physisorption (duration: $2 \mathrm{~h}$ ) and afterwards GAG solution was replaced by EDC crosslinking solution (duration: $2 \mathrm{~h}$ ). Binding of fluorescently labeled GAG was investigated after $1 \mathrm{~d}$ by CLSM ('+' indicates successful binding, '-' indicates no binding detected). Intact network structures were verified by imaging of unlabeled collagen fibrils using the reflection signal of cLSM

$\begin{array}{lllll}\text { (1) GAG solution (during physisorption) } & \mathrm{pH} 5 & \mathrm{pH} & \mathrm{pH} \mathrm{7.4} & \mathrm{pH} \mathrm{7.4} \\ \text { (2) EDC reaction } & \mathrm{pH} 5 & \mathrm{pH} 6 & \mathrm{pH} \mathrm{5} \\ \text { HA } & + & - & - & - \\ \text { hSHA } & + & + & + & -\end{array}$


$\mathrm{pH} 7.4$, as subsequent crosslinking at $\mathrm{pH} 5$ would have been successful in case HA had been physisorbed at $\mathrm{pH}$ 7.4. prior to crosslinking.) Lowering $\mathrm{pH}$ led to a strong increase of HA binding during physisorption, whereas binding of hsHA was not affected. A pH lower than 5 was omitted due to the known instability of Coll I fibrils at highly acidic conditions. ${ }^{16}$ Next we could show covalent binding using EDC to be essential for a stable Coll I/HA modification (Table 2 and Fig. 4A). Without covalent binding a drastic decrease of network bound HA was observed during the first day.

Additionally, we verified a homogeneous GAG distribution throughout the whole Coll I network layer for HA and hsHA (Fig. 3). A homogeneous distribution of GAGs was also observed for networks with other pore sizes and mechanics (not investigated in further detail herein).

The approach of GAG physisorption at pH 5 and subsequent EDC crosslinking was further evaluated using fluorimetry in order to determine GAG content and stability at physiological buffer condition. As expected from the initial experiments hsHA was stably bound to the Coll I networks via physisorption or covalent linking via EDC at an amount of approx. $30 \mu \mathrm{g}$ per mg Coll I (Fig. 4A). For HA, stable binding was only observed after covalent binding via EDC, while physisorption led to a gradual loss of HA over time. Interestingly, the GAG/Coll I binding ability at $\mathrm{pH} 5$ with subsequent EDC crosslinking was not affected by the degree of sulfation as similar amounts of stably bound GAG (approx. $30 \mu \mathrm{g}$ per $\mathrm{mg}$ Coll I) were observed for HA and hsHA. We also verified an unchanged network topology after GAG binding by imaging GAG/Coll I networks using cLSM (Fig. 4B-D).

Our results show that stable binding of GAGs with and without sulfate groups to Coll I fibrils can be achieved via EDC crosslinking. For HA, crosslinking was necessary, and binding was found to be strongly dependent on $\mathrm{pH}$. A low $\mathrm{pH}$ was required during incubation of the $\mathrm{HA}$ solution for the initial physisorption of HA to Coll I. Otherwise no binding was observed even with the following EDC reaction performed at optimal conditions ( $\mathrm{pH} 5-6^{45}$ ). Binding of hsHA was almost independent of the tested $\mathrm{pH}$ conditions during initial physisorption and subsequent crosslinking. Consequently, intermolecular interactions between GAGs and Coll I during initial physisorption are concluded to play the major role for successful binding, whereas EDC crosslinking enables permanent fixation of the physisorbed amount of HA.
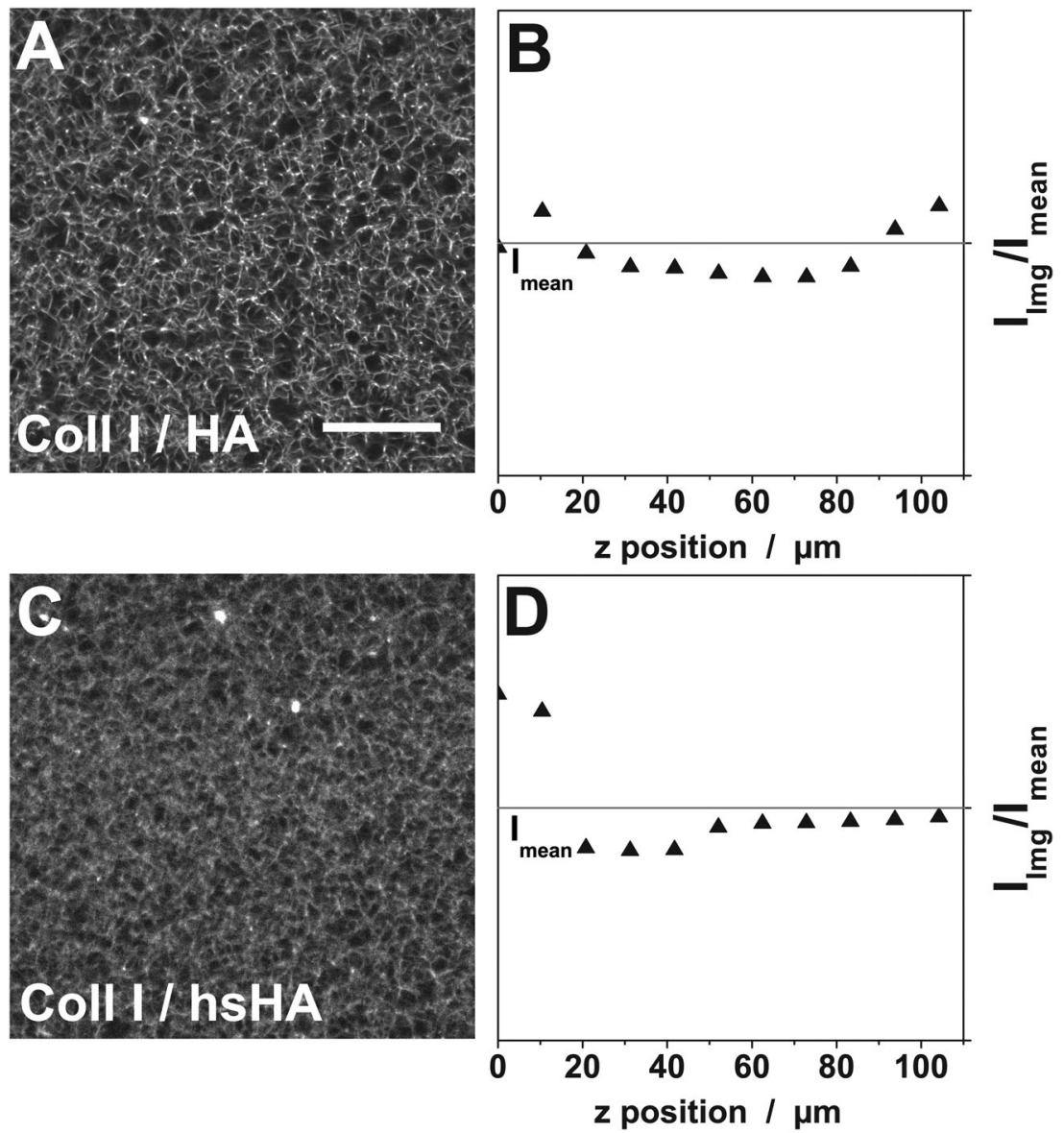

Fig. 3 Distribution of covalently bound HA and hsHA in Coll I networks ( $c_{\text {Colll }}=2 \mathrm{mg} \mathrm{ml}^{-1}$, pore size $\sim 2 \mu \mathrm{m}$ ). (A-C) cLSM image of fluorescently labeled $\mathrm{HA} / \mathrm{hsHA}$ in the Coll I network in $x y$-plane. Scale bar: $25 \mu \mathrm{m}$. (B-D) Distribution of fluorescently labeled HA/hsHA in z-direction determined from exemplary CLSM image stacks ( $/$ Img - fluorescence intensity of individual images, $I_{\text {mean }}$ - mean intensity of the whole stack). Stacks were composed of $x y$-images every $10 \mu \mathrm{m}$ over a distance of $100 \mu \mathrm{m}$ (total network thickness: $150 \mu \mathrm{m}$ ). 
A
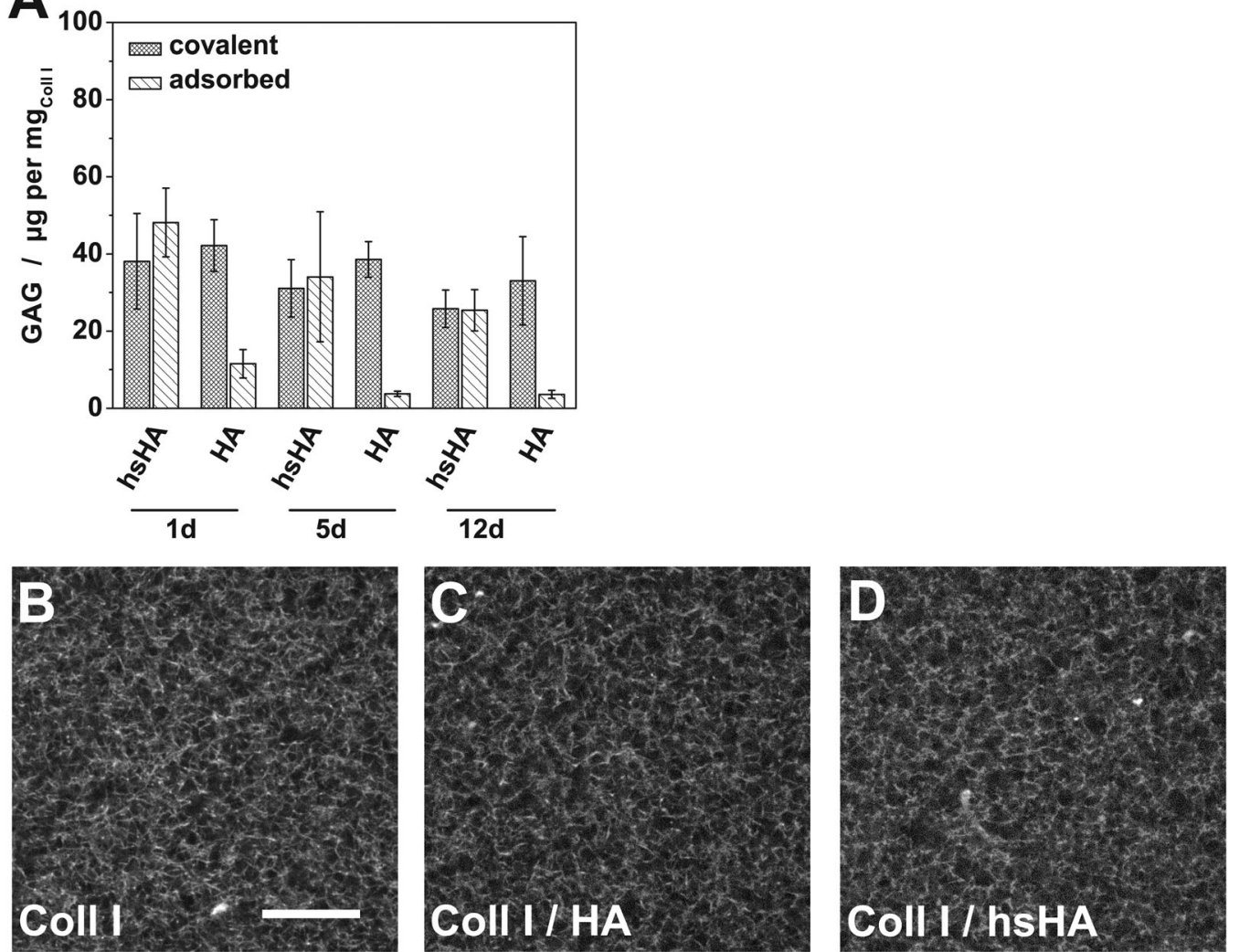

Fig. 4 Characterization of Coll I/GAG networks ( $c_{\text {colll }}=2 \mathrm{mg} \mathrm{ml}^{-1}$ ). (A) GAG quantification after $1 \mathrm{~d}$ (in PBS), $5 \mathrm{~d}$ and $12 \mathrm{~d}$ (in PBS with $1 \%$ BSA) via fluorimetry. GAG amounts were normalized to Coll I amount within the matrices $(40 \mu \mathrm{g})$ as determined previously. ${ }^{39}$ Error bars indicate \pm standard deviation (S.D.). Measurements were done in duplicate in three independent experiments $(n=3)$. (B-D) cLSM images of the microstructure of unmodified and GAG modified Coll I networks. Scale bar: $25 \mu \mathrm{m}$.

The relevant intermolecular interactions between GAGs and Coll I during initial physisorption are thought to depend on the net charge of the molecules. Our experiments were performed at $\mathrm{pH}$ values between 5 and 7.4 and the number of positive charges along the Coll I fibril should be higher at low $\mathrm{pH}^{51}$ (Isoelectric point (IEP) of Coll $\mathrm{I}$ is reported to be between 5 and $8^{52-54}$ and depends on the ionic strength of the solvent. ${ }^{53}$ ) Consequently, electrostatic attraction between Coll I and the negatively charged GAGs should increase at acidic conditions and explain the stronger interaction and initial physisorption of HA to Coll I at pH 5. In contrast, similar binding of hsHA was observed at pH 5 and 7.4. This finding can be explained by the high density of sulfate groups of hsHA. At pH 7.4 hsHA exhibits a high negative charge density in contrast to the much lower charge density of $\mathrm{HA}^{55}$ Hence, there is strong electrostatic interaction between hsHA and Coll I, with negligible $\mathrm{pH}$ sensitivity within the tested range.

In essence, two issues have to be considered when explaining the differential binding of HA and hsHA to Coll I in dependence on $\mathrm{pH}$. The positive net charge of Coll $\mathrm{I}$ is higher at $\mathrm{pH} 5$ than at $\mathrm{pH}$ 7.4. Carboxyl groups of HA should be mostly deprotonated at pH 5 ( $\mathrm{p} K_{\mathrm{a}}$ of HA carboxyl groups is reported to be around 3-4 ${ }^{26,52}$ ) hence, HA should exhibit a negative net charge sufficient for strong electrostatic attraction to Coll I. At $\mathrm{pH} 7.4$ the positive net charge of Coll $\mathrm{I}$ is much lower than at pH 5 and only for the highly sulfated GAG (hsHA) the much more negative net charge is high enough to still allow for a strong interaction with Coll I.

The efficiency of the subsequent covalent binding via EDC is also expected to depend on $\mathrm{pH}$. EDC reacts with protonated carboxyl groups and the protonation of the carboxyl groups increases at acidic $\mathrm{pH}$, which should positively affect the reaction. The observation that binding of hsHA was unaffected by changing pH can be explained by two effects. Firstly, due to the strong electrostatic interaction between Coll I and hsHA at low and high $\mathrm{pH}$, covalent binding is not required for a stable hsHA binding. Secondly, the covalent binding of hsHA is weakly $\mathrm{pH}$ dependent because more carboxyl groups are already protonated at $\mathrm{pH}$ 7.4. It is reported that the $\mathrm{p} K_{\mathrm{a}}$ of the carboxyl groups of sulfated GAGs rises with an increasing number of sulfate groups. ${ }^{55}$ This means that covalent binding of HA is again more dependent on $\mathrm{pH}$ and acidic conditions are required in contrast to hsHA.

\section{Conclusion}

Defined biomimetic microenvironments with adjustable parameters are necessary for a detailed understanding of cell-matrix 
interactions in in vitro studies. Our results show that 3D fibrillar matrices based on Coll I can be prepared with defined topology and mechanics and can be further modified with sulfated and non-sulfated GAGs, leaving the network structure unaffected. Importantly, while sulfated GAGs can be stably attached to Coll I networks via physisorption or covalent binding at neutral and acidic conditions, non-sulfated HA requires acidic conditions and covalent binding for stable attachment.

The presented approach circumvents known problems that can occur when adding GAGs during Coll I fibril formation. ${ }^{17,56}$ It allows to maintain Coll I fibril and network structure, independent of GAG modification. Hence, Coll I matrices can be made available with defined topology, different mechanics and optional GAG functionalization in a combinatorial manner. This will allow for a better distinction of the impact of GAG functionalization on cell behavior in topologically and mechanically defined matrices. ${ }^{1,22,57}$

\section{Acknowledgements}

The authors acknowledge the support of grants from ESF 'European Social Funds' and Free State of Saxony (SAB, grant: 100147954) to TP and KF, from Human Frontier Science Program Organization (HFSPO, grant: RGP0051/2011) to TP and from Deutsche Forschungsgemeinschaft (DFG, grant: SFB-TR67/B10, Z3 and INST 268/293-1 FUGG) to LK, TP and MS. Fruitful discussions with Michael Ansorge are gratefully acknowledged.

\section{References}

1 P. A. Janmey and D. A. Weitz, Trends Biochem. Sci., 2004, 29, 364-370.

2 M. A. Schwartz and D. W. DeSimone, Curr. Opin. Cell Biol., 2008, 20, 551-556.

3 F. Rosso, A. Giordano, M. Barbarisi and A. Barbarisi, J. Cell. Physiol., 2004, 199, 174-180.

4 M. Aumailley and B. Gayraud, J. Mol. Med., 1998, 76, 253-265. 5 J. C. Adams and F. M. Watt, Development, 1993, 117, 1183-1198.

6 A. E. X. Brown and D. E. Discher, Curr. Biol., 2009, 19, R781-789.

7 L. G. Griffith and M. A. Swartz, Nat. Rev. Mol. Cell Biol., 2006, 7, 211-224.

8 J. A. Green and K. M. Yamada, Adv. Drug Delivery Rev., 2007, 59, 1293-1298.

9 E. Cukierman, R. Pankov, D. R. Stevens and K. M. Yamada, Science, 2001, 294, 1708-1712.

10 M. W. Tibbitt and K. S. Anseth, Biotechnol. Bioeng., 2009, 103, 655-663.

11 J. A. M. Ramshaw, Y. Y. Peng, V. Glattauer and J. A. Werkmeister, J. Mater. Sci.: Mater. Med., 2008, 20, 3-8.

12 K. Wolf, S. Alexander, V. Schacht, L. M. Coussens, U. H. von Andrian, J. van Rheenen, E. Deryugina and P. Friedl, Semin. Cell Dev. Biol., 2009, 20, 931-941.

13 M. P. Lutolf and J. A. Hubbell, Nat. Biotechnol., 2005, 23, 47-55.
14 F. Gobeaux, G. Mosser, A. Anglo, P. Panine, P. Davidson, M.-M. Giraud-Guille and E. Belamie, J. Mol. Biol., 2008, 376, 1509-1522.

15 J. R. Harris, A. Soliakov and R. J. Lewis, Micron, 2013, 49, 60-68.

16 Y. Li, A. Asadi, M. R. Monroe and E. P. Douglas, Mater. Sci. Eng., C, 2009, 29, 1643-1649.

17 D. R. Stamov and T. Pompe, Soft Matter, 2012, 8, 10200.

18 I. K. Piechocka, A. S. G. van Oosten, R. G. M. Breuls and G. H. Koenderink, Biomacromolecules, 2011, 12, 2797-2805.

19 J. Sapudom, S. Rubner, S. Martin, T. Kurth, S. Riedel, C. T. Mierke and T. Pompe, Biomaterials, 2015, 52, 367-375.

20 J. M. Lee, H. H. L. Edwards, C. A. Pereira and S. I. Samii, J. Mater. Sci.: Mater. Med., 1996, 7, 531-541.

21 Y.-J. Hwang, J. Granelli and J. Lyubovitsky, ACS Appl. Mater. Interfaces, 2012, 4, 261-267.

22 L. Schaefer and R. M. Schaefer, Cell Tissue Res., 2010, 339, 237-246.

23 D. Nikitovic, M. Tzardi, A. Berdiaki, A. Tsatsakis and G. N. Tzanakakis, Inflammation, 2015, 6, 169.

24 E. H. Knelson, J. C. Nee and G. C. Blobe, Trends Biochem. Sci., 2014, 39, 277-288.

25 S. Misra, V. C. Hascall, R. R. Markwald and S. Ghatak, Inflammation, 2015, 201.

26 T. C. Laurent and J. R. Fraser, FASEB J., 1992, 6, 2397-2404.

27 J. R. Fraser, T. C. Laurent and U. B. Laurent, J. Intern. Med., 1997, 242, 27-33.

28 K. T. Dicker, L. A. Gurski, S. Pradhan-Bhatt, R. L. Witt, M. C. Farach-Carson and X. Jia, Acta Biomater., 2014, 10, 1558-1570.

29 M. Kataropoulou, C. Henderson and H. Grant, Hum. Exp. Toxicol., 2003, 22, 65-71.

30 Y. Yang, C. Sun, M. E. Wilhelm, L. J. Fox, J. Zhu and L. J. Kaufman, Biomaterials, 2011, 32, 7932-7940.

31 A. van der Smissen, V. Hintze, D. Scharnweber, S. Moeller, M. Schnabelrauch, A. Majok, J. C. Simon and U. Anderegg, Biomaterials, 2011, 32, 8938-8946.

32 D. Scharnweber, L. Hübner, S. Rother, U. Hempel, U. Anderegg, S. A. Samsonov, M. T. Pisabarro, L. Hofbauer, M. Schnabelrauch, S. Franz, J. Simon and V. Hintze, J. Mater. Sci.: Mater. Med., 2015, 26, DOI: 10.1007/s10856-015-5563-7.

33 F. J. O’Brien, B. A. Harley, I. V. Yannas and L. J. Gibson, Biomaterials, 2005, 26, 433-441.

34 D. Stamov, M. Grimmer, K. Salchert, T. Pompe and C. Werner, Biomaterials, 2008, 29, 1-14.

35 A. Miron, S. Rother, L. Huebner, U. Hempel, I. Käppler, S. Moeller, M. Schnabelrauch, D. Scharnweber and V. Hintze, Macromol. Biosci., 2014, 14, 1783-1794.

36 K. Salchert, U. Streller, T. Pompe, N. Herold, M. Grimmer and C. Werner, Biomacromolecules, 2004, 5, 1340-1350.

37 V. Hintze, S. Moeller, M. Schnabelrauch, S. Bierbaum, M. Viola, H. Worch and D. Scharnweber, Biomacromolecules, 2009, 10, 3290-3297.

38 T. Pompe, S. Zschoche, N. Herold, K. Salchert, M.-F. Gouzy, C. Sperling and C. Werner, Biomacromolecules, 2003, 4, 1072-1079. 
39 K. Franke, J. Sapudom, L. Kalbitzer, U. Anderegg and T. Pompe, Acta Biomater., 2014, 10, 2693-2702.

40 J. L. Hutter and J. Bechhoefer, Rev. Sci. Instrum., 1993, 64, 1868-1873.

41 L. J. Kaufman, C. P. Brangwynne, K. E. Kasza, E. Filippidi, V. D. Gordon, T. S. Deisboeck and D. A. Weitz, Biophys. J., 2005, 89, 635-650.

42 D. C. Morse, Macromolecules, 1998, 31, 7044-7067.

43 F. C. MacKintosh, J. Käs and P. A. Janmey, Phys. Rev. Lett., 1995, 75, 4425-4428.

44 N. A. Kurniawan, L. H. Wong and R. Rajagopalan, Biomacromolecules, 2012, 13, 691-698.

45 Pierce Biotechnology, Thermo Scientific User Guide: EDC, https://tools.thermofisher.com/content/sfs/manuals/ MAN0011256_EDC_UG.pdf (26.10.2015).

46 G. T. Hermanson, Bioconjugate Techniques, Elsevier, 2nd edn, 2008.

47 C. Yang, Bull. Mater. Sci., 2012, 35, 913-918.

48 Z. Grabarek and J. Gergely, Anal. Biochem., 1990, 185, 131-135.

49 G. R. Kunkel, M. Mehrabian and H. G. Martinson, Mol. Cell. Biochem., 1981, 34, 3-13.

50 D. R. Stamov, T. A. Khoa Nguyen, H. M. Evans, T. Pfohl, C. Werner and T. Pompe, Biomaterials, 2011, 32, 7444-7453.
51 J. Zhang, B. Senger, D. Vautier, C. Picart, P. Schaaf, J.-C. Voegel and P. Lavalle, Biomaterials, 2005, 26, 3353-3361.

52 M. Luescher, M. Rüegg and P. Schindler, Biopolymers, 1974, 13, 2489-2503.

53 U. Freudenberg, S. H. Behrens, P. B. Welzel, M. Müller, M. Grimmer, K. Salchert, T. Taeger, K. Schmidt, W. Pompe and C. Werner, Biophys. J., 2007, 92, 2108-2119.

54 N. Barbani, L. Lazzeri, C. Cristallini, M. G. Cascone, G. Polacco and G. Pizzirani, J. Appl. Polym. Sci., 1999, 72, 971-976.

55 A. Zieris, R. Dockhorn, A. Röhrich, R. Zimmermann, M. Müller, P. B. Welzel, M. V. Tsurkan, J.-U. Sommer, U. Freudenberg and C. Werner, Biomacromolecules, 2014, 15, 4439-4446.

56 B. Stadlinger, V. Hintze, S. Bierbaum, S. Möller, M. C. Schulz, R. Mai, E. Kuhlisch, S. Heinemann, D. Scharnweber, M. Schnabelrauch and U. Eckelt, J. Biomed. Mater. Res., Part B, 2012, 100, 331-341.

57 A. Chopra, M. E. Murray, F. J. Byfield, M. G. Mendez, R. Halleluyan, D. J. Restle, D. Raz-Ben Aroush, P. A. Galie, K. Pogoda, R. Bucki, C. Marcinkiewicz, G. D. Prestwich, T. I. Zarembinski, C. S. Chen, E. Puré, J. Y. Kresh and P. A. Janmey, Biomaterials, 2014, 35, 71-82. 